\title{
Spontaneous symmetry breaking in the laser transition
}

\author{
P. Gartner \\ Centre International de Formation et de Recherche Avancées en Physique \\ - National Institute of Materials Physics, Bucharest-Măgurele, Romanid用
}

(Dated: December 19, 2018)

\begin{abstract}
In analogy with equilibrium phase transitions, we address the problem of the instability to symmetry-breaking perturbations of systems undergoing a laser transition. The symmetry in question is the $U(1)$ invariance with respect to a phase factor, and the perturbation is a coherent field $E$, coupled to the exciton. At the rate equation level we analyze first the case of a cavity containing a single, two-level emitter, and then a chain of such cavities interacting by photon hopping processes. In both cases spontaneous symmetry breaking takes place when the system is in the lasing phase. For the laser transition, the analogue of the thermodynamic limit is the scaling limit of vanishing cavity loss and light-matter coupling, $\kappa \rightarrow 0, g \rightarrow 0$, so that $g^{2} / \kappa$ remains finite. We show that in the lasing regime anomalous averages persist in the $E \rightarrow 0$ limit, provided that the scaling limit is performed first. Lasing diagnosis based on robust anomalous averages is compared numerically with the familiar coherence criterion $g^{(2)}(0)=1$, and the advantages of the former are discussed.
\end{abstract}

PACS numbers: 42.55.Ah, 42.50.Ct, 42.55.Sa, 78.67.Hc

\section{INTRODUCTION}

The lasers were recognized quite early $\underline{1}^{\underline{\underline{-3}}}$ as examples of systems undergoing a phase transition in conditions of nonequilibrium. The lasing regime plays the role of the 'ordered' phase, separated form the 'normal' one by a critical point, the laser threshold. The latter is usually identified by a jump in the population of the resonant cavity mode as a function of pumping. In a log$\log$ plot this jump in the input-output curve is given by $\ln \beta, \underline{\underline{4}}$ where the $\beta$-factor is the fraction of photons spontaneously emitted into the lasing mode.

The advent of nanolasers, with few emitters and wellconfined photon modes, allowed for $\beta$-factors close to unity, erasing the intensity jump in the so-called 'thresholdless' lasers ${ }^{5-8}$. Consequently, alternative lasing criteria have been proposed, not always agreeing with each other. Some are still based on the photon number $n$, by requiring $n>1^{9,10}$. Also, coherence tests, probing the Poissonian photon statistics are usually applied, the most frequently invoked being the requirement for the second order auto-correlation function at zero delay, $g^{(2)}(0)$, to be close to unity $11-14$. Other criteria have been considered as well 11 . The issue is still debated 15 and has even prompted Nature Photonics to publish a 'checklist' 16 to provide a certain level of confidence in identifying lasing.

It is therefore surprising that a common feature of equilibrium phase transitions, namely the spontaneous symmetry breaking was largely ignored. The instability against symmetry-reducing perturbation is a characteristic of the ordered phase, and in this paper we address the problem of an analogue behavior in the lasing regime.

We consider first the case of a single, two-level emitter interacting resonantly with a cavity mode. Various equivalent terminologies are used in the literature for the two levels, which may be seen as two atomic configurations, a qubit, a spin, or an electron-hole pair in a quantum dot which can form an exciton or recombine. The symme- try of this problem is the invariance with respect to an arbitrary common phase factor assigned to the photon and exciton quasi-spin operators. A coherent excitation field $E$, coupled only to the excitonic degree of freedom breaks this symmetry, and generates anomalous averages, i.e. expectation values that are strictly zero in the symmetric case. The 'spontaneous' nature of the symmetry breaking is defined as the persistence of the anomalous averages in the limit of zero perturbation, as in the case of spontaneous magnetization in magnetic systems.

The analysis requires that the conditions for a sharp phase transitions are met. Rice and Carmichae $\underline{1}^{4}$ have drawn the attention to the role of a certain limit in ensuring an abrupt transition, with a well-defined threshold point, in the same way as the thermodynamic limit is a necessary condition in the equilibrium theory. For the problem considered here it was shown ${ }^{17}$ that the appropriate limit is obtained by scaling down to zero both the cavity losses $\kappa$ and the Jaynes-Cummings (JC) coupling $g$ so that the ratio $g^{2} / \kappa$ remains finite. The order of limits is crucial, the scaling limit should be performed before taking $E \rightarrow 0$. We show that in this case the survival of the anomalous averages takes place in the parameter domain corresponding to the lasing regime, and only there.

Obviously, such statements assume a proof by analytic methods. This is made possible by treating the system at the rate equation level, which is the analog of meanfield theories in equilibrium phase transitions, and which reduces the infinite hierarchy of equations of motion to a closed system for a limited set of expectation values. The treatment is a standard approach in quantum optics textbooks $\frac{18,19}{19}$ and, as far as the laser transition is concerned, it was shown to produce exact results 17 . In the context of masers pumped by random injection 20,21 symmetry breaking was also discussed in a mean-field setting $\underline{3}$.

A second model addressed in the paper consists of an array of optical cavities each in interaction with a two- 
level emitter and coupled to its nearest neighbors by photon hopping 22 . This leads to a photonic energy band of extended Bloch states. Applying the same procedure as above we show spontaneous symmetry breaking taking place in the Bloch mode resonant with the exciton, which is also the lasing mode.

The paper is organized as follows: In both cases, after describing the models, the laser transition is first analyzed in the symmetric case, and the role of the scaling limit in obtaining a sharp transition is proven. Then the changes due to the symmetry-breaking seed are introduced. In the single cavity case numerical illustrations are also presented, showing that robustness of the anomalous averages is seen even before actually reaching the prescribed limits, but signaling when we are in their proximity. In this respect, the numerical examples show that the behavior of anomalous averages confirm the $g^{(2)}(0)$ criterion of lasing. This is important, since the former are easily accesible at the rate-equation level, while the latter is not.

\section{SINGLE TWO-LEVEL EMITTER IN A CAVITY}

In this section we analyze the case of a two-level emitter in resonance with a cavity mode. The emitter-photon interaction is described using the familiar JC Hamiltonian. Dissipative effects, like cavity losses and spontaneous decay of the exciton are also considered in the Lindblad formalism. The system is excited incoherently by an up-scattering Lindblad term. This problem was extensively studied in the literature, both in atomic 23,24 and in semiconductor quantum dot in a cavity contexts $25-28$.

As an additional feature, we include in the Hamiltonian a symmetry-breaking seed in the form of an infinitesimal coherent pumping, represented by the dipole coupling of the exciton to a resonant electric field.

In the rotating frame the Hamiltonian reads $(\hbar=1$ throughout the paper)

$$
H=g b^{\dagger} \sigma+g^{*} b \sigma^{\dagger}+E^{*} \sigma+E \sigma^{\dagger} .
$$

Here $b^{\dagger}, b$ are the operators of the photon mode, $\sigma^{\dagger}, \sigma$ are the pseudo-spin raising and lowering operators for the two-level system, $g$ is the JC coupling constant and $E$ is the strength of the coherent excitation.

The equation of motion (EOM) for an arbitrary operator $A$ consists of a coherent, von Neuman part and of the incoherent Lindblad term contribution

$$
\begin{aligned}
\frac{d}{d t}\langle A\rangle= & -i\langle[A, H]\rangle \\
& +\sum_{\alpha} \frac{\mu_{\alpha}}{2}\left\langle\left[L_{\alpha}^{\dagger}, A\right] L_{\alpha}+L_{\alpha}^{\dagger}\left[A, L_{\alpha}\right]\right\rangle,
\end{aligned}
$$

where $\mu_{\alpha}$ is the rate associated with the scattering process defined by the operator $L_{\alpha}$. Three such processes are considered: the spontaneous relaxation of the atom (or excitonic loss in quantum dot language) with $\mu_{\alpha}$ denoted by $\gamma$ and $L_{\alpha}=\sigma$, the cavity losses with the rate $\kappa$ and operator $b$, and the pumping simulated as an upscattering process, $L_{\alpha}=\sigma^{\dagger}$, with the rate $P$.

For $E=0$ the theory is $U(1)$-invariant, i.e. it is insensitive to an arbitrary phase factor attached to the elementary operators $e^{i \lambda} b, e^{i \lambda} \sigma$. In other words the expectation values do not depend on $\lambda$ and therefore non-zero averages should appear only when the operators are combined in phase-independent expressions, like $\left\langle b^{\dagger} \sigma\right\rangle,\left\langle b^{\dagger} b\right\rangle$ a.s.o. In terms of the Glauber-Sudarshan $\mathcal{P}$-representation 29 this is related to the rotation invariance in the complex plane of the photonic quasi-distribution function $\mathcal{P}$. The phase symmetry is broken by the coherent excitation, which imposes its own phase on the system, and brings in anomalous averages, like $\langle b\rangle,\left\langle b^{\dagger}\right\rangle$ and $\langle\sigma\rangle,\left\langle\sigma^{\dagger}\right\rangle$ As in the equilibrium phase-transition theory, by 'spontaneous' it is understood that anomalous averages remain nonzero even in the limit $E \rightarrow 0$. If and when this takes place is the subject of what follows.

Applying Eq.(2) one obtains the EOM for the normal averages as

$$
\begin{aligned}
\frac{d}{d t}\left\langle b^{\dagger} b\right\rangle= & -i g\left\langle b^{\dagger} \sigma\right\rangle+i g^{*}\left\langle b \sigma^{\dagger}\right\rangle-\kappa\left\langle b^{\dagger} b\right\rangle \\
\frac{d}{d t}\left\langle\sigma^{\dagger} \sigma\right\rangle= & i g\left\langle b^{\dagger} \sigma\right\rangle-i g^{*}\left\langle b \sigma^{\dagger}\right\rangle-\gamma\left\langle\sigma^{\dagger} \sigma\right\rangle+P\left\langle\sigma \sigma^{\dagger}\right\rangle \\
& +i E^{*}\langle\sigma\rangle-i E\left\langle\sigma^{\dagger}\right\rangle \\
\frac{d}{d t}\left\langle b^{\dagger} \sigma\right\rangle= & i g^{*}\left\langle b^{\dagger} b\left(\sigma^{\dagger} \sigma-\sigma \sigma^{\dagger}\right)\right\rangle+i g^{*}\left\langle\sigma^{\dagger} \sigma\right\rangle \\
- & \frac{P+\gamma+\kappa}{2}\left\langle b^{\dagger} \sigma\right\rangle+i E\left\langle b^{\dagger}\left(\sigma^{\dagger} \sigma-\sigma \sigma^{\dagger}\right)\right\rangle
\end{aligned}
$$

It is seen that in Eqs. (3) the anomalous averages are brought in by the symmetry-breaking excitation. For them one derives the following EOM, driven by the perturbation

$$
\begin{aligned}
\frac{d}{d t}\langle b\rangle & =-i g\langle\sigma\rangle-\frac{\kappa}{2}\langle b\rangle, \\
\frac{d}{d t}\langle\sigma\rangle & =i g^{*}\left\langle b\left(\sigma^{\dagger} \sigma-\sigma \sigma^{\dagger}\right)\right\rangle \\
& -\frac{P+\gamma}{2}\langle\sigma\rangle+i E\left\langle\sigma^{\dagger} \sigma-\sigma \sigma^{\dagger}\right\rangle .
\end{aligned}
$$

These equations represent the starting of an infinite hierachy of EOM. They are transformed into a closed system by applying a factorization approximation to the expectation values. The most popular is the rate equation formalism, which is essentially a mean-field approach to our many-body problem, and is widely used in the literature 30.31 .

To be specific, in Eq. (3c) one factorizes level occupancies and photon operators in separate averages

$$
\left\langle b^{\dagger} b\left(\sigma^{\dagger} \sigma-\sigma \sigma^{\dagger}\right)\right\rangle \approx\left\langle b^{\dagger} b\right\rangle\left(\left\langle\sigma^{\dagger} \sigma\right\rangle-\left\langle\sigma \sigma^{\dagger}\right\rangle\right) .
$$


Similarly, in Eqs.(3c) and (4b) one uses

$$
\left\langle b\left(\sigma^{\dagger} \sigma-\sigma \sigma^{\dagger}\right)\right\rangle \approx\langle b\rangle\left(\left\langle\sigma^{\dagger} \sigma\right\rangle-\left\langle\sigma \sigma^{\dagger}\right\rangle\right) .
$$

It is not immediately obvious why other factorizations, like $\left\langle b^{\dagger} \sigma\right\rangle \approx\left\langle b^{\dagger}\right\rangle\langle\sigma\rangle$ are not kept. In symmetric theories the anomalous averages involved simply do not appear, but now they are driven by the $E$-excitation, and in principle could contribute.

The answer is provided by the cluster expansion theory ${ }^{32,33}$. With averages of the elementary raising and lowering operators as singlets, the rate-equation limit is the systematic truncation at the doublet level. The following facts have to be considered: (i) Triplets and quadruplets are neglected. For instance, unfactorized $\left\langle b^{\dagger} \sigma^{\dagger} \sigma\right\rangle$ are not kept. (ii) In the factorization of the quantities appearing in the LHS of Eqs.(5), (6), all terms in which the operators $\sigma^{\dagger}$ and $\sigma$ are separated in different averages cancel out because of the difference involving the $\sigma^{\dagger} \sigma$ and the $\sigma \sigma^{\dagger}$ contributions. (iii) The remaining terms are of the form

$$
\left\langle b^{\dagger} b \sigma^{\dagger} \sigma\right\rangle \approx \delta\left\langle b^{\dagger} b\right\rangle\left\langle\sigma^{\dagger} \sigma\right\rangle+\left\langle b^{\dagger}\right\rangle\langle b\rangle\left\langle\sigma^{\dagger} \sigma\right\rangle .
$$

Inserting the definition $\delta\left\langle b^{\dagger} b\right\rangle=\left\langle b^{\dagger} b\right\rangle-\left\langle b^{\dagger}\right\rangle\langle b\rangle$ one obtains the factorization shown in Eq.(5). A similar argument works for Eq. (6).

As a result one is left with a closed system of rate equations for the following unknowns: the photon number $n=$ $\left\langle b^{\dagger} b\right\rangle$, the upper level (or excitonic) occupancy $f=\left\langle\sigma^{\dagger} \sigma\right\rangle$, the photon-assisted polarization $\psi=-i g\left\langle b^{\dagger} \sigma\right\rangle$ and the anomalous averages $\alpha=g^{*}\langle b\rangle$ and $\varphi=-i\langle\sigma\rangle$. The lower level occupancy is then $1-f=\left\langle\sigma \sigma^{\dagger}\right\rangle$. The system reads

$$
\begin{aligned}
& \frac{d}{d t} n=2 \psi_{1}-\kappa n \\
& \frac{d}{d t} f=-2 \psi_{1}-\Gamma f+P-E^{*} \varphi-E \varphi^{*} \\
& \frac{d}{d t} \psi=|g|^{2} n(2 f-1)+|g|^{2} f-\frac{\Gamma^{\prime}}{2} \psi+E \alpha^{*}(2 f-1) \\
& \frac{d}{d t} \alpha=|g|^{2} \varphi-\frac{\kappa}{2} \alpha \\
& \frac{d}{d t} \varphi=\alpha(2 f-1)-\frac{\Gamma}{2} \varphi+E(2 f-1)
\end{aligned}
$$

Here we denoted by $\psi_{1}$ the real part of $\psi, \Gamma=P+\gamma$ and $\Gamma^{\prime}=P+\gamma+\kappa$. Before analyzing the solution in the limit $E \rightarrow 0$, we summarize below the $E=0$ situation.

\section{A. The laser transition in the scaling limit}

In the absence of the symmetry-breaking term one is left with the first three Eqs.(8), for the unknowns $n, f$ and $\psi$. In the steady state the time derivatives are vanishing and the system becomes algebraic. One notices that $\psi$ becomes real, $\psi_{1}=\psi$, and is given by Eq. (8c) as

$$
2 \psi=R^{\prime} n(2 f-1)+R^{\prime} f,
$$

with $R^{\prime}=4|g|^{2} / \Gamma^{\prime}$. We use Eq. $2 \psi=\kappa n$, and stay with the main variables, the photon and the upper-level populations.

$$
\begin{aligned}
& \kappa n=R^{\prime} n(2 f-1)+R^{\prime} f, \\
& \kappa n=-\Gamma f+P .
\end{aligned}
$$

The first of these equation is simply the photon balance between the loss through the cavity walls and the net generation, consisting of spontaneous and stimulated emission $R^{\prime}(n+1) f$ minus absorption $R^{\prime} n(1-f)$, as in the theory of Einstein coefficients ${ }^{18}$, with $R^{\prime}$ identified as the spontaneous emission rate. The condition can be recast in the form

$$
f_{L}^{\prime}-f=\frac{f}{2 n} \quad \text { with } \quad f_{L}^{\prime}=\frac{1}{2}+\frac{\kappa}{2 R^{\prime}} .
$$

It is easy to see that $f_{L}^{\prime}$ is the population for which the gain in the active medium (stimulated emission minus absorption) exactly compansated the cavity losses. One consequence of Eq.111) is that $f_{L}^{\prime}$ is an upper bound for the solution, $f \leqslant f_{L}^{\prime}$ for all pump values.

The second equation is again a balance condition, expressing the fact that in the steady state the loss of an excitation, either photonic $\kappa n$, or excitonic $\gamma f$, should be compensated by the pumping process $P(1-f)$. The condition can be rewritten as

$$
f_{N}-f=\frac{\kappa n}{\Gamma} \quad \text { with } \quad f_{N}=\frac{P}{P+\gamma}
$$

where $f_{N}$ represents the excitonic occupancy of the isolated emitter $(g=0)$. Again one obtains an upper bound $f \leqslant f_{N}$ for all $P$.

By multiplying the Eqs. (11) and (12) one obtains a quadratic equation for the exciton population

$$
\left(f_{N}-f\right)\left(f_{L}^{\prime}-f\right)=\frac{\kappa}{2(P+\gamma)} f
$$

whose lower solution $f<\min \left\{f_{N}, f_{L}^{\prime}\right\}$ is the physical one. One notices that for small values of $\kappa$ the RHS of this equation becomes small, and this pushes the solution close to either one of the limit cases, $f_{N}$, or the other, $f_{L}^{\prime}$, whichever is lower (see Fig.11). Therefore, with increasing $P$, the system changes between two regimes, characterized by either $f \approx f_{N}$ or $f \approx f_{L}^{\prime}$. As long as the RHS of Eq. (13) is nonzero the transition is smooth, with no well-defined threshold.

In order to obtain an abrupt transition, with precise transition points, one has to perform some limiting procedure. The obvious limit is $\kappa \rightarrow 0$, which brings the RHS of the Eq.13) to zero, and thus leads the exciton population as function of pumping to develop angular points, as it shifts from one limit solution to the other. On the other hand, in order to preserve the qualitative picture of Fig 1 one simultaneously takes the limit $g \rightarrow 0$ so 


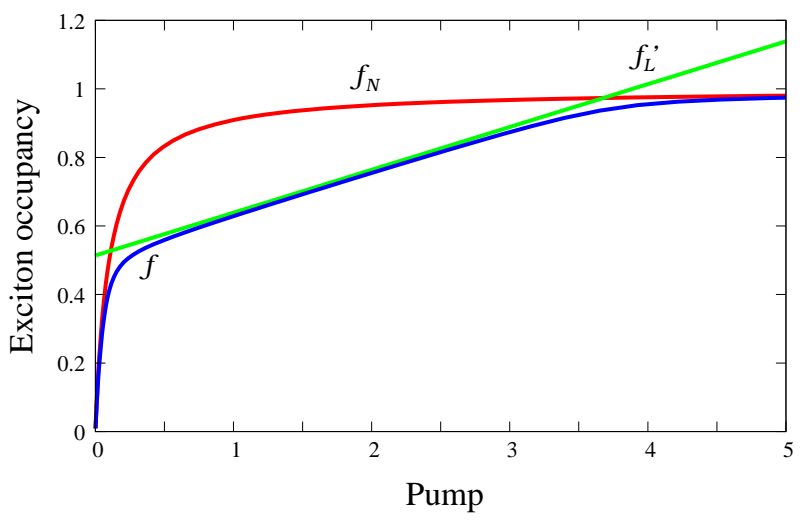

Figure 1. The limit solutions $f_{N}$ (red) and $f_{L}^{\prime}$ (green) are upper bounds for the true one (blue). The parameters used are $\gamma=0.1, \kappa=0.01$ and $g=0.1$.

that $g^{2} / \kappa$ remains finite. This amounts to only a minor change in $f_{L}^{\prime}$, which becomes now

$$
f_{L}^{\prime} \rightarrow f_{L}=\frac{1}{2}+\frac{\kappa}{2 R}=\frac{1}{2}+\frac{\kappa(P+\gamma)}{8|g|^{2}}
$$

where the limit of $\kappa / R^{\prime}$ is $\kappa / R$, with $R=4|g|^{2} / \Gamma=$ $4|g|^{2} /(P+\gamma)$. Without the $g \rightarrow 0$ limit one would have the trivial result $f_{L}^{\prime} \rightarrow 1 / 2$. It is worth noting that the $\beta$-factor is proportional to $|g|^{2}$, and therefore this can be also viewed as a small $\beta$ limit, as required for a sharp transition ${ }^{4}$.

The conclusion is that in the limit in which both $g$ and $\kappa$ are scaled down to zero, while keeping the proportion $g^{2} / \kappa$ finite, one has a sharp transition between $f=f_{N}$ and $f=f_{L}$. The non-analyticity of the solution is represented by angular points appearing at the $f_{N}=f_{L}$ crossing points.

Turning now to the photon population, we have from Eq.(12)

$$
\kappa n=(P+\gamma)\left(f_{N}-f\right) .
$$

Again, two contrasting situations appear. In the $f=$ $f_{N}$ regime it is clear that the value of photon number cannot prevent the product $\kappa n$ to vanish in the scaling limit. This is the normal, non-lasing phase (hence the index N). On the contrary, in the case $f=f_{L}$ the photon number goes to infinity, such that the product $\kappa n$ stays finite. This is indicative of "an explosion of stimulated emission" index L).

Note that the condition for the existence of real roots $P$ for the quadratic equation $f_{N}=f_{L}$ is $|g|^{2} / \kappa>2 \gamma \underline{17}$. This is the lasing condition upon the parameters. When it is met there are two crossing points. Going from low to high pump values the system is initially normal, up to the first crossover. This is the threshold value $P_{t h}$, where the system starts lasing. Reaching the second crossing it becomes normal again, due to the so-called quenching phenomenon ${ }^{24}$, which is a consequence of the excitationinduced dephasing.

In the absence of a size parameter one cannot define the thermodynamic limit for our problem, as in the theory of equilibrium phase transition. This role is played here by the scaling limit. In this case a "macroscopic" photon population corresponds to one that grows like $1 / \kappa$. It might seem trivial that $n$ increases when the cavity quality is getting better, but one should keep in mind that in the scaling limit the rate of photon generation, $R \sim|g|^{2}$, is reduced too, and precisely in the same ratio as the loss rate.

Before closing this discussion we note that the proof of these results can be made rigorous, not depending on the rate-equation approximation 17 .

\section{B. Spontaneous symmetry breaking for the two-level laser}

In the presence of the symmetry-breaking excitation, Eqs. (8d) and (8ed) for the anomalous averages are driven by the $E$-term. Solving for steady-state values of $\alpha$ and $\varphi$ gives

$$
\begin{aligned}
& \alpha=E \frac{f-\frac{1}{2}}{f_{L}-f}, \\
& \varphi=E \frac{\kappa}{2|g|^{2}} \frac{f-\frac{1}{2}}{f_{L}-f} .
\end{aligned}
$$

The anomalous averages are driven by $E$, their phases are the phase of $E$ and can be easily factored out. Therefore, and for the sake of simplicity, we will assume that $E$ is real and positive.

It is already clear that these averages may survive in the limit $E \rightarrow 0$ only if simultaneously $f \rightarrow f_{L}$, in a way that keeps the ratio at a finite value. We have seen that in the scaling limit, and in the lasing regime, $f$ does approach $f_{L}$. It remains to analyze the interplay of the two limit procedures.

Introducing these results in Eqs. (8a)-(8c), one notices again that $\psi$ is real, obeying now the relation

$$
2 \psi=\kappa n=R^{\prime} n(2 f-1)+R^{\prime} f+\frac{8 E^{2}}{\Gamma^{\prime}} \frac{\left(f-\frac{1}{2}\right)^{2}}{f_{L}-f},
$$

which is the extension of Eq.110a , and can be recast as

$$
\kappa n\left(f_{L}^{\prime}-f\right)=\frac{\kappa}{2} f+E^{2} \frac{\kappa}{|g|^{2}} \frac{\left(f-\frac{1}{2}\right)^{2}}{f_{L}-f} .
$$

The generalization of Eq.(10b) reads

$$
\kappa n=(P+\gamma)\left(f_{N}-f\right)-E^{2} \frac{\kappa}{|g|^{2}} \frac{f-\frac{1}{2}}{f_{L}-f} .
$$

Now it is easy to eliminate $n$ and one obtains for $f$ the 
cubic equation

$$
\begin{array}{r}
{\left[\left(f_{N}-f\right)\left(f_{L}^{\prime}-f\right)-\frac{\kappa}{2(P+\gamma)} f\right]\left(f_{L}-f\right)} \\
-E^{2} \frac{\kappa^{2} \Gamma^{\prime}}{8|g|^{4} \Gamma}(f-1 / 2)=0
\end{array}
$$

where the quadratic polynomial in the square brackets, denoted in what follows by $Q(f)$, provides the roots for the symmetric problem, see Eq.(13).

The natural question arising in the presence of two limit procedures concerns their order. We will show below that performing first the limit $E \rightarrow 0$, the anomalous averages go to zero and one recovers the symmetric case results of Section IIA Applying subsequently the scaling limit one reaches a sharp laser transition, as described there. This is the expected behavior, but the proof requires some attention. On the contrary, if the scaling limit is performed first, the anomalous averages remain non-zero even after the $E \rightarrow 0$ limit, and this spontaneous symmetry breaking takes place in the lasing regime. Below we analyze the two limit orders.

(i) If the $E \rightarrow 0$ limit is performed first, Eq.(20) becomes $Q(f)\left(f_{L}-f\right)=0$. We analyze first possibility is that its solution is the lower root of $Q(f)=0$, as in the symmetric case. We know that this root obeys $f<f_{N}, f_{L}^{\prime}$, but in order to prove that the anomalous averages disappear indeed, one has to show that $f$ stays away from $f_{L}$. This is not immediate, since $f_{L}$ too, like $f$, is on the lower side of $f_{L}^{\prime}$. We prove that actually $f$ is strictly smaller than $f_{L}$ by checking that $Q\left(f_{L}\right)$ is negative. Indeed, using the explicit expressions of $f_{N}, f_{L}^{\prime}$ and $f_{L}$ one obtains

$$
\begin{aligned}
Q\left(f_{L}\right) & =\left(\frac{P-\gamma}{2(P+\gamma)}-\frac{\kappa(P+\gamma)}{8|g|^{2}}\right) \frac{\kappa^{2}}{8|g|^{2}} \\
& -\frac{\kappa}{2(P+\gamma)}\left(\frac{1}{2}+\frac{\kappa(P+\gamma)}{8|g|^{2}}\right) .
\end{aligned}
$$

Here one notices that the only positive contribution comes from the first term, proportional to $P$, which is exactly cancelled by the last $P$-term. All the other terms being negative one has $Q(f)<0$, which proves that $f_{L}$ is placed between the roots, i.e. $f<f_{L}$.

A second possibility to be considered is that, in the limit $E \rightarrow 0$, the physical solution converges to $f_{L}$. This can be ruled out by noticing from Eq.(20) that in such a case $f_{L}-f \sim E^{2}$ and then the anomalous average $\varphi$ would go to infinity. But this is impossible, since $\varphi$ is the expectation value of $\sigma$, a bounded operator.

(ii) If the scaling limit is performed first, Eq.(20) becomes

$$
\left(f_{N}-f\right)\left(f_{L}-f\right)^{2}-E^{2} \frac{\kappa^{2}}{8|g|^{4}}(f-1 / 2)=0,
$$

After that, in the $E \rightarrow 0$ limit, one possibility is $f \rightarrow f_{N}$, which is the normal phase. But in the lasing regime we see that $f$ converges to $f_{L}$ in such a way that $f_{L}-f \sim E$,
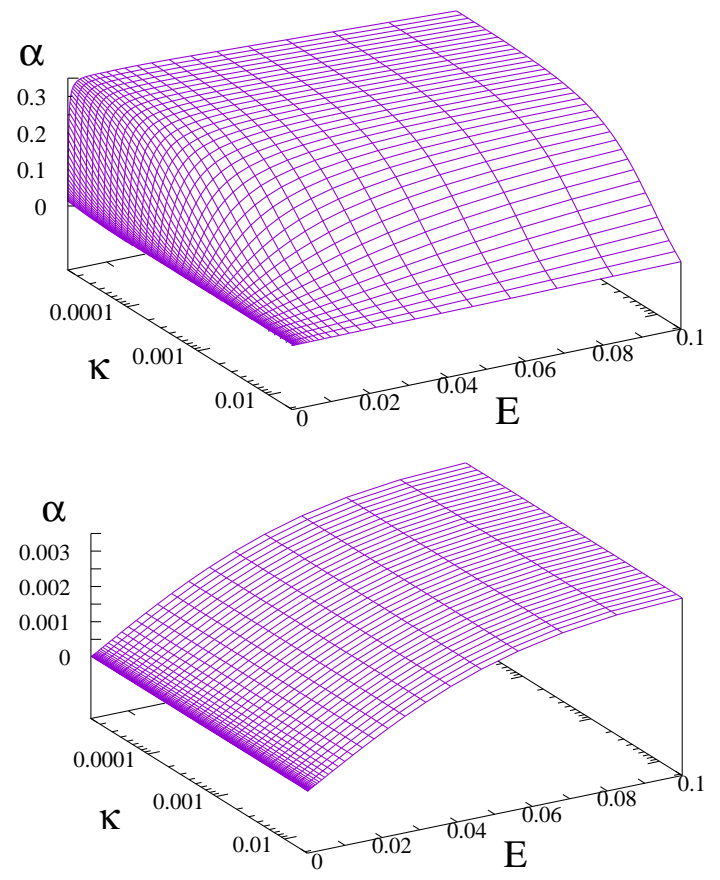

Figure 2. The anomalous average $\alpha$ as function of the symmetry-breaking seed $E$, for different values of $\kappa$. Other parameters: $g^{2}=\kappa, P=0.3, \gamma=0.02$ (upper panel) and $g^{2}=\kappa / 200, P=0.3, \gamma=0.01$ (lower panel).

which is exactly the order in the infinitesimal parameter that keeps the anomalous averages non-zero.

This statement is the main result of the paper: spontaneous symmetry breaking does take place, but only in the lasing phase as it is defined by the scaling limit. Additionally, the result stresses that the scaling limit is instrumental in understanding the laser transition.

The photon population behavior in the scaling limit is contained in Eq.(18), which has now the form

$$
\kappa n=E^{2} \frac{\kappa}{|g|^{2}} \frac{\left(f-\frac{1}{2}\right)^{2}}{\left(f_{L}-f\right)^{2}}=\frac{\kappa}{|g|^{2}} \alpha^{2},
$$

which amounts to $\left\langle b^{\dagger} b\right\rangle=|\langle b\rangle|^{2}$. This is what is heuristically expected from a coherent photonic state, in which the $b$ operator behaves like a c-number.

From a practical point of view the limits discussed above cannot be reached numerically, and even less experimentally. Nevertheless, one may bring the parameters in the asymptotic domain sufficiently close to the limits to see their influence, in the sense of predicting with good accuracy the behavior of the system. The correct limit order is simulated by taking $\kappa, g^{2} \ll E$.

Indeed, as seen in Fig 2, upper panel, the anomalous average $\alpha$ goes to zero with $E$, but the convergence gets significantly delayed by decreasing $\kappa$ and $g^{2}$, as a numerical hint that the scaling limit prevents the vanishing of $\alpha$ altogether. This is the situation for parameters corresponding to the laser regime. On the contrary, in the lower panel the parameters do not meet the lasing condi- 

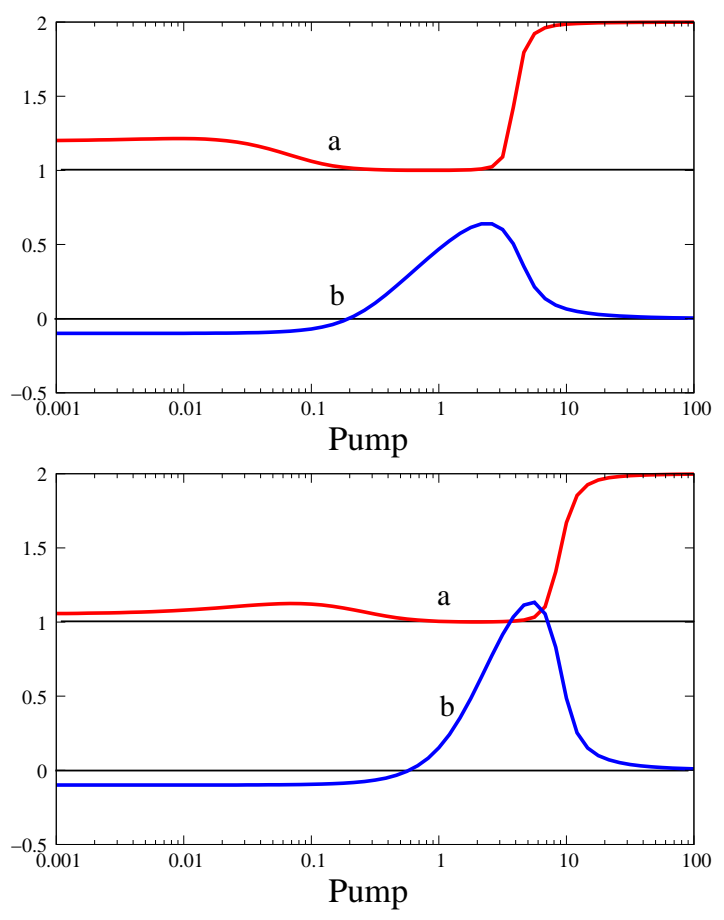

Figure 3. (a) $g^{(2)}(0)$ and (b) $\alpha$, as functions of pumping. The parameters are: $\kappa=0.01, g=0.1, \gamma=0.02$ for the upper panel and $\kappa=0.04, g=0.3, \gamma=0.05$ for the lower one. In both $E=0.1$. Horizontal lines at 1 and 0 are guide to the eye.

tion and one sees that the decrease of $\alpha$ with $E$ is completely insensitive to the $\kappa$ values. Note also the absolute values, which are two orders of magnitude lower.

It is also worth noting that $\alpha$ shows a positive bump in the pumping interval in which the photon autocorrelation function at zero time delay, $g^{(2)}(0)$, is close to unity. The latter is currently used as a test of coherent light, but unfortunately it is not accessible at the rate equation level, being related to the quadruplet $\left\langle b^{\dagger} b^{\dagger} b b\right\rangle$. For instance, in the cases illustrated in Fig 3 in order to calculate $g^{(2)}(0)$ we had to solve the full, infinite hierarchy by using a continued fraction method 17,23 . In contrast the anomalous average $\alpha \sim\langle b\rangle$ is a singlet, and thus it was easy to obtain.

The situations shown in Fig 3 are relatively far from $E=0$, and in the lower panel further away from the scaling limit too. Yet both cases show that the examination of $\alpha$ is a useful tool in its own right for signaling the lasing behavior. One should also keep in mind that $g^{(2)}(0)=1$ is a necessary but not sufficient condition for coherent light.

\section{CAVITY ARRAYS}

In this section we show that the analysis above and its conclusions are not limited to the simple case of a single, two-level emitter. Here we consider the more complicated system of a linear array of cavities, coupled by a photon hopping term, describing the possible leaking from one cavity to its neigbors. A two-level emitter is placed in each of these cavities and is coupled to its photon mode by the JC interaction.

The Hamiltonian of such a system is

$$
\begin{aligned}
H & =\omega \sum_{r} b_{r}^{\dagger} b_{r}+J \sum_{r}\left[b_{r}^{\dagger} b_{r+1}+b_{r+1}^{\dagger} b_{r}\right] \\
& +\varepsilon \sum_{r} \sigma_{r}^{\dagger} \sigma_{r}+\sum_{r}\left[g b_{r}^{\dagger} \sigma_{r}+g^{*} b_{r} \sigma_{r}^{\dagger}\right] .
\end{aligned}
$$

The chain of $N$ cavities is assumed homogeneous, with the photon frequency $\omega$ and the exciton energy $\varepsilon$ independent on the position $r$ on the chain. The same holds for the JC coupling $g$ and for the hopping term $J$ to nearest neigbors. Assuming cyclic boundary conditions and taking advantage of the translation invariance, the photon part of the Hamiltonian becomes diagonal in the plane-wave representation

$$
H_{p h}=\sum_{k} \omega_{k} b_{k}^{\dagger} b_{k},
$$

where $\omega_{k}=\omega+2 J \cos k$ are the Bloch-mode frequencies, forming the energy band of photonic eigenstates. The momentum $k$ runs on the Brillouin zone (BZ), represented by the interval $(-\pi, \pi]$. The corresponding operators are connected to the local ones by

$$
b_{k}=\frac{1}{\sqrt{N}} \sum_{r} e^{i k r} b_{r}, \text { and } b_{r}=\frac{1}{\sqrt{N}} \sum_{k} e^{-i k r} b_{k} .
$$

To the above Hamiltonian a symmetry-breaking seed is added in the form

$$
H_{s b}=\sum_{r}\left[E_{r}^{*}(t) \sigma_{r}+E_{r}(t) \sigma_{r}^{\dagger}\right]
$$

where $E_{r}(t)=E(t) e^{i q r}$, is chosen to excite a particular Bloch mode $q$. The translation invariance is spoiled by this $r$-dependence, but it is formally recovered by the unitary transform

$$
\sigma_{r} \rightarrow e^{i q r} \sigma_{r}, \quad b_{r} \rightarrow e^{i q r} b_{r}
$$

which restores the expression of the translation invariant Hamiltonian up to a shift of the photon spectrum

$$
H_{p h}=\sum_{k} \omega_{k-q} b_{k}^{\dagger} b_{k} .
$$

In the rotating frame with respect to

$$
H_{0}=\varepsilon \sum_{k} b_{k}^{\dagger} b_{k}+\varepsilon \sum_{r} \sigma_{r}^{\dagger} \sigma_{r},
$$

and considering a resonant coherent excitation $E(t)=$ $E e^{-i \varepsilon t}$, one is left with the Hamiltonian

$$
\begin{aligned}
H= & -\sum_{k} \Delta_{k-q} b_{k}^{\dagger} b_{k}+\sum_{r, k}\left[g_{r}(k) b_{k}^{\dagger} \sigma_{r}+g_{r}^{*}(k) b_{k} \sigma_{r}^{\dagger}\right] \\
& +\sum_{r}\left[E^{*} \sigma_{r}+E \sigma_{r}^{\dagger}\right],
\end{aligned}
$$


where $\Delta_{k}=\varepsilon-\omega_{k}$ is the $k$-mode detuning and $g_{r}(k)=$ $g e^{i k r} / \sqrt{N}$. The $k, r$ double summation could be avoided by defining $\sigma_{k}$ as the Fourier transform of $\sigma_{r}$, but this is not as useful for what follows as Eq. (26) because of the more complicated commutation relations ensuing.

The dissipative part of the model consists of including at each position $r$ on the chain Lindblad terms identical to those described in Eq.(2). For instance, for the cavity loss one has

$$
\frac{d}{d t}\langle A\rangle_{\text {cav.loss }}=\frac{\kappa}{2} \sum_{r}\left\langle\left[b_{r}^{\dagger}, A\right] b_{r}+b_{r}^{\dagger}\left[A, b_{r}\right]\right\rangle,
$$

which, by unitarity, has the same expression in the $k$ representation.

The EOM for this system, considered at the rate equation level involves a limited set of expectation values. We have the photon Bloch-mode populations, which is the Fourier transform of the photon-photon correlation along the chain

$$
n_{k}=\left\langle b_{k}^{\dagger} b_{k}\right\rangle=\sum_{r} e^{-i k r}\left\langle b_{r}^{\dagger} b_{0}\right\rangle .
$$

We made use here of the translation invariance property $\left\langle b_{r}^{\dagger} b_{r^{\prime}}\right\rangle=\left\langle b_{r-r^{\prime}}^{\dagger} b_{0}\right\rangle$. The inverse relation reads

$$
\left\langle b_{r}^{\dagger} b_{0}\right\rangle=\frac{1}{N} \sum_{k} e^{i k r} n_{k}
$$

from which one obtains the on-site population as a BZ average of the mode population

$$
n=\left\langle b_{0}^{\dagger} b_{0}\right\rangle=\frac{1}{N} \sum_{k} n_{k}
$$

Again, by translation invariance, the on-site population is the same on all sites, and therefore its notation does not carry an index.

Similar quantities arise in connection with the excitonic populations and correlations

$$
\begin{aligned}
& f_{k}=\left\langle\sigma_{k}^{\dagger} \sigma_{k}\right\rangle=\sum_{r} e^{-i k r}\left\langle\sigma_{r}^{\dagger} \sigma_{0}\right\rangle \\
& \left\langle\sigma_{r}^{\dagger} \sigma_{0}\right\rangle=\frac{1}{N} \sum_{k} e^{i k r} f_{k}
\end{aligned}
$$

and the on-site exciton population $f=\left\langle\sigma_{0}^{\dagger} \sigma_{0}\right\rangle$ is obtained as the BZ average of $f_{k}$. It is obvious from the definitions that $n_{k}$ and $f_{k}$ are real, positive quantities. In a previous paper $^{22}$ the $\sigma-\sigma$ correlators were neglected, so that in this respect the present treatment is slightly more general.

One also encounters mixed, photon-exciton correlators, and it is convenient to define, in analogy with the single cavity case

$$
\psi_{k}=-i g \sqrt{N}\left\langle b_{k}^{\dagger} \sigma_{0}\right\rangle
$$

The site $r=0$ does not play a special role since, by translation invariance one has $\left\langle b_{k}^{\dagger} \sigma_{r}\right\rangle=e^{-i k r}\left\langle b_{k}^{\dagger} \sigma_{0}\right\rangle$.
The anomalous averages one has to consider are

$$
\begin{aligned}
& \alpha_{k}=g^{*} \sqrt{N}\left\langle b_{k}\right\rangle=g^{*}\left\langle b_{r}\right\rangle N \delta_{k, 0}=\alpha N \delta_{k, 0}, \\
& \varphi_{k}=-i \sqrt{N}\left\langle\sigma_{k}\right\rangle=-i\left\langle\sigma_{r}\right\rangle N \delta_{k, 0}=\varphi N \delta_{k, 0} .
\end{aligned}
$$

The $r$-dependence is spurious, the sites being identical. This brings the $k$-depenence to a $\delta$-function located at $k=0$. As before, the indexless $\alpha, \varphi$ denote the BZ averages of the respective $k$-dependent quantities. Note that $N \delta_{k, 0}$ becomes $2 \pi \delta(k)$ in the infinite chain limit.

With these notations the EOM have the form

$$
\begin{aligned}
\frac{d}{d t} n_{k}= & 2 \psi_{k, 1}-\kappa n_{k}, \\
\frac{d}{d t} f_{k}= & P-\Gamma f_{k}+2 \psi_{k, 1}(2 f-1)-2 \psi_{1} 2 f \\
& +\left(E^{*} \varphi_{k}+\text { c.c. }\right)(2 f-1)-\left(E^{*} \varphi+\text { c.c. }\right) 2 f \\
\frac{d}{d t} \psi_{k}= & -\left(\frac{\Gamma^{\prime}}{2}+i \Delta_{k-q}\right) \psi_{k}+|g|^{2} n_{k}(2 f-1) \\
& +|g|^{2} f_{k}+E \alpha_{k}^{*}(2 f-1) .
\end{aligned}
$$

The subindex 1 in $\psi_{k, 1}$ and $\psi_{1}$ denotes the real part, the absence of the momentum index implies the BZ average, and c.c. is short for complex conjugate. Similitudes and differences to Eqs. (3) are obvious. The nonlinear terms stem from factorizations of the same kind as used there, but slightly more complicated. For instance we split populations from photon-assisted polarizations in separate factors, i.e.

$$
\left\langle b_{k} \sigma_{r}^{\dagger}\left[\sigma_{0}, \sigma_{0}^{\dagger}\right]\right\rangle \approx\left\langle b_{k} \sigma_{r}^{\dagger}\right\rangle\left\langle\left[\sigma_{0}, \sigma_{0}^{\dagger}\right]\right\rangle=e^{i k r}\left\langle b_{k} \sigma_{0}^{\dagger}\right\rangle(1-2 f),
$$

for $r \neq 0$, but one has exactly $\left\langle b_{k} \sigma_{r}^{\dagger}\left[\sigma_{0}, \sigma_{0}^{\dagger}\right]\right\rangle=\left\langle b_{k} \sigma_{0}^{\dagger}\right\rangle$ if $r=0$. These single-site terms lead to the $k$-independent subtractions appearing in Eq.41b. The EOM for the anomalous averages read

$$
\begin{aligned}
\frac{d}{d t} \alpha_{k} & =-\left(\frac{\kappa}{2}-i \Delta_{k-q}\right) \alpha_{k}+|g|^{2} \varphi_{k} \\
\frac{d}{d t} \varphi_{k} & =-\frac{\Gamma}{2} \varphi_{k}+\alpha_{k}(2 f-1)+E(2 f-1) N \delta_{k, 0}
\end{aligned}
$$

\section{A. Laser transition in cavity arrays}

As in the single cavity case, we analyze first the laser transition in the absence of symmetry breaking. To this end we consider the steady-state solution of Eqs. (41) for $E=0$. It is easy to eliminate $\psi_{k, 1}$ using the first equation: $2 \psi_{k, 1}=\kappa n_{k}$. As a consequence one has also $2 \psi_{1}=\kappa n$. From Eq. (41b) one obtains succesively

$$
f_{k}=f_{N}+(2 f-1) \frac{\kappa}{P+\gamma} n_{k}-2 f \frac{\kappa}{P+\gamma} n,
$$

then, after averaging over the BZ

$$
\frac{\kappa}{P+\gamma} n=f_{N}-f
$$


which is used to rewrite Eq.(44) as

$$
f_{k}=(2 f-1) \frac{\kappa}{P+\gamma} n_{k}+\left[f_{N}-2 f\left(f_{N}-f\right)\right] .
$$

This is a first equation connecting the populations $f_{k}$ and $n_{k}$. The term in square brackets is quadratic in $f$ and in what follows will be denoted by $\Phi(f)$ or just $\Phi$. It is important to note that $\Phi(f)$ remains a positive quantity for any $f$.

The relation (45) is the analogue of Eq. (12) and translates the fact that the Hamiltonian conserves the total excitation number $\sum_{r}\left[b_{r}^{\dagger} b_{r}+\sigma_{r}^{\dagger} \sigma_{r}\right]$, which is influenced only by the dissipation terms. Also, an important consequence is that one recovers the same upper bound $f \leqslant f_{N}$.

A second equation is derived from Eq.(41c) and reads

$$
\kappa n_{k}=(2 f-1) R_{k}^{\prime} n_{k}+R_{k}^{\prime} f_{k},
$$

where $R_{k}^{\prime}=4|g|^{2} \Gamma^{\prime} /\left(\Gamma^{\prime 2}+4 \Delta_{k}^{2}\right)$ denotes the spontaneous transition rate into mode $k$, depending on its detuning $\Delta_{k}$. This is the counterpart of Eq.10a). Now one can eliminate $f_{k}$ from the two equations and obtain $n_{k}$ as a function of $f$ only. The result can be put in the form

$$
n_{k}=\frac{\frac{|g|^{2}}{\kappa} \Gamma^{\prime} \Phi(f)}{\Delta_{k}^{2}+2 \frac{|g|^{2}}{\kappa} \frac{\Gamma^{\prime 2}}{\Gamma}\left(f_{L}-f\right)},
$$

in which, in the denominator, the detuning term was separated. Then, by averaging the result over the BZ and using again Eq. (45), we obtain a closed equation for $f$. In the limit of large $N$ the BZ averaging is expressed by a Lorentzian integral with respect to the detuning

$$
f_{N}-f=|g|^{2} \frac{1}{2 \pi} \int_{-\pi}^{\pi} \frac{A}{(2 \cos k-\Delta)^{2}+\delta^{2}} d k .
$$

Here $\Delta=(\varepsilon-\omega) / J$ is the on-site detuning normalized to the hopping rate $J$ and $A=\left(\Gamma^{\prime} \Phi\right) /\left(J^{2} \Gamma\right)$. Also

$$
\delta=\frac{\Gamma^{\prime}}{J} \sqrt{\frac{2|g|^{2}}{\kappa \Gamma}} \sqrt{f_{L}-f}=B \sqrt{f_{L}-f} .
$$

The notation used for the $\delta^{2}$ term in the denominator of Eq. (49) suggests that the quantity is positive, which is by no means obvious. Nevertheless, the positivity of the Bloch-mode populations $n_{k}$ entails the positivity of the denominator in Eq. (48) for all $k$. If additionally we assume that there are resonant modes in the system, $\Delta_{k}=0$ for some $k$, then $\delta^{2}$ must be positive indeed. This is the case we consider from now on. Then $\delta$ is real and moreover, by this argument we also recover the second upper bound $f \leqslant f_{L}$.

Eq.(49) can be solved only numerically, since the unknown function $f$ is expressed as an integral involving other $f$-dependent quantities, like $\Phi$ and $\delta$. Still, the behavior of the solution in the scaling limit is analytically accessible. The constants $A$ and $B$ are stable (neither vanishing, nor divergent) in this limit since $g$ and $\kappa$ appear in the scaling ratio $g^{2} / \kappa$ and $\Gamma^{\prime} \rightarrow \Gamma$. Only the prefactor $|g|^{2}$ is not compensated, and seems to imply that one has necessarily $f \rightarrow f_{N}$. But this contradicts the inequality $f \leqslant f_{L}$, when $f_{L}<f_{N}$.

The solution of the paradox relies on the possibility that the integral itself becomes divergent, as indeed is the case, due to the existence of resonant modes, and provided $\delta$ also vanishes, i.e. $f \rightarrow f_{L}$ in the scaling limit.

This heuristic argument can be made precise by performing the integral analytically ${ }^{22}$, which is done by mapping the $[-\pi, \pi]$ interval over the unit cercle in the complex plane and using the residue theorem. The result is

$$
\left(f_{N}-f\right) \sqrt{f_{L}-f}=|g|^{2} \frac{A}{B} \Re\left\{\frac{i}{\zeta_{2}-\zeta_{1}}\right\},
$$

where $\zeta_{1,2}$ are the roots of the quadratic equation $z^{2}-$ $u z+1=0$, with $u=\Delta+i \delta$, obeying $\left|\zeta_{1}\right|<1<\left|\zeta_{2}\right|$.

The conclusion is again that in the scaling limit $f$ converges to either $f_{N}$ or $f_{L}$, whichever is the smaller. As the pumping increases, the transition between these options is sharp, as in the single emitter case. The macroscopic photon population occurs only in the $f=f_{L}$ regime. It is now obvious that the macroscopically occupied modes are the resonant ones.

As a final remark, we note that the obvious main difference from Eq.(13) appears in the reduced power of $f_{L}-f$. This is a consequence of the integration over $k$, which reduces the singularity $1 / \delta^{2}$ of the integrand to $1 / \delta$. The factor $i /\left(\zeta_{2}-\zeta_{1}\right)$, related to the photonic density of states, introduces additional singularities only if the resonant mode is at the spectral edge. We assume for simplicity that we are not in such special situations. Otherwise, the divergence of the density of states at the band edges modifies the power of $f_{L}-f$, but does not change the conclusion.

\section{B. Spontaneous symmetry breaking in cavity arrays}

By solving the system of Eqs.(43) for the anomalous averages one obtains in the steady state

$$
\begin{aligned}
\alpha_{k} & =\frac{f-1 / 2}{f_{L}-f-i \frac{\Gamma}{4|g|^{2}} \Delta_{q}} E N \delta_{k, 0}, \\
\varphi_{k} & =\frac{\kappa-2 i \Delta_{q}}{2|g|^{2}} \frac{f-1 / 2}{f_{L}-f-i \frac{\Gamma}{4|g|^{2}} \Delta_{q}} E N \delta_{k, 0},
\end{aligned}
$$

where we used the fact that the photon spectrum is even $\Delta_{-q}=\Delta_{q}$. It is clear that the survival of these quantities in the $E \rightarrow 0$ limit is possible only if the symmetrybreaking field is chosen to excite a resonant mode, i.e. 
$\Delta_{q}=0$. In this case we get the simpler forms

$$
\begin{aligned}
\alpha_{k} & =\frac{f-1 / 2}{f_{L}-f} E N \delta_{k, 0}, \\
\varphi_{k} & =\frac{\kappa}{2|g|^{2}} \frac{f-1 / 2}{f_{L}-f} E N \delta_{k, 0},
\end{aligned}
$$

Moreover $f$ has to converge to $f_{L}$ as the first power of the field. In order to prove this statement we now solve Eqs.(41) including the contribution of the anomalous averages.

By proceeding exactly as in Section $11 \mathrm{~A}$ one obtains the symmetry-broken version of Eq. (49)

$$
\begin{gathered}
f_{N}-f=|g|^{2} \frac{1}{2 \pi} \int_{-\pi}^{\pi} \frac{A}{(2 \cos (k-q)-\Delta)^{2}+\delta^{2}} d k \\
+\frac{\kappa}{|g|^{2}} \frac{(f-1 / 2)^{2}}{\left(f_{L}-f\right)^{2}} E^{2} .
\end{gathered}
$$

Again, one can consider $E$ real, without loss of generality. The shift by $q$, introduced by the space modulation of the coherent exciting field, is irrelevant for the result, since it appears in a periodic function integrated over a whole period. Integrating as above one is left with

$$
\begin{gathered}
f_{N}-f=|g|^{2} \frac{A}{B \sqrt{f_{L}-f}} \Re\left\{\frac{i}{\zeta_{2}-\zeta_{1}}\right\} \\
+\frac{\kappa}{|g|^{2}} \frac{(f-1 / 2)^{2}}{\left(f_{L}-f\right)^{2}} E^{2} .
\end{gathered}
$$

It is obvious now that performing first the $E \rightarrow 0$ limit is killing both $\alpha_{k}$ and $\varphi_{k}$, since $f$ differs from $f_{L}$ as long as $g \neq 0$. After that one is left with the situation described in section

On the contrary, if the scaling limit is taken first, it cancels the first term in Eq. (55) and with $f \neq f_{N}$ one concludes that in the lasing regime $f_{L}-f \sim E$, when $E$ goes subsequently to zero. This is precisely the condition for the persistence of anomalous averages as the symmetry-breaking seed goes to zero.

\section{CONCLUSIONS}

We have proven the instability of the laser systems to symmetry-breaking infinitesimal perturbations. In this respect the laser transition is similar to equilibrium phase transitions. Due to the invariance with respect to phase rotation, the averages like $\langle b\rangle$ or $\langle\sigma\rangle$ have no preferred direction in the complex plane and therefore must be zero. Non-zero values are generated by a symmetry-breaking seed and we have shown that they persist when the seed is removed. Such spontaneous symmetry breaking takes place when the system is in the lasing phase, and only then.

We discussed first the case of a single, two-level emitter embedded in a cavity and interacting resonantly with its photon mode. Second, we considered a chain of such cavities, allowing for photon hopping between nearest neighbors. In both cases the symmetry-breaking perturbation was a coherent field $E$, in resonant dipole coupling with the exciton.

At the level of the rate equation we showed analytically that the mechanism of the spontaneous symmetry breaking is connected to the existence of a sharp laser transition, with a well-defined threshold point. This involves the scaling limit $\kappa \rightarrow 0$ and $g \rightarrow 0$, so that $g^{2} / \kappa$ remains finite. It was shown that the correct limit order is scaling limit first, followed by $E \rightarrow 0$. In the case of cavity arrays, the symmetry breaking occurs in the resonant Bloch mode, which is also the lasing mode.

Numerical estimates have shown that the sensitivity to symmetry-breaking perturbations develops and manifests itself even before reaching these limits. Anomalous averages remain high if the conditions for lasing are met, but drop fast with the perturbation otherwise. In this sense such averages can be used as evidence for lasing. For instance, numerical examples showed that a large value of $\langle b\rangle$ confirms the $g^{2}(0) \approx 1$ test. The former has the advantage that $\langle b\rangle$, as a singlet, is accesible at the rate-equation level, while $g^{2}(0)$, which contains a quadruplet, is not.

\section{ACKNOWLEDGMENT}

The author acknowledges financial support from CNCS-UEFISCDI Grant No. PN-III-P4-ID-PCE-20160221.
* gartner2@gmail.com

1 R. Graham and H. Haken, Z. Physik 237, 31 (1970).

2 S. Grossmann and P. H. Richter, Z. Physik 242, 458 (1971).

3 V. DeGiorgio and M. O. Scully,
Phys. Rev. A 2,1170 (1970)
${ }^{4}$ P. R. Rice and H. J. Carmichael, Phys. Rev. A 50, 4318 (1994)
H. Yokoyama and S. D. Brorson, J. Appl. Phys. 66, 4801 (1989)

6 M. Khajavikhan, A. Simic, M. M. Katz, J. H. Lee, B. Slutsky, A. Mizrahi, V. Lomakin, and Y. Fainman, Nature 482, 204 (2012) 
7 F. De Martini and G. R. Jacobovitz, Phys. Rev. Lett. 60, 1711 (1988).

8 Y. Ota, M. Kakuda, K. Watanabe, S. Iwamoto, and Y. Arakawa, Opt. Express 25, 19981 (2017)

${ }^{9}$ G. Björk, A. Karlsson, and Y. Yamamoto, Phys. Rev. A 50, 1675 (1994)

10 A. Auffèves, D. Gerace, J.-M. Gérard, M. França Santos, L. C. Andreani, and J.-P. Poizat, Phys. Rev. B 81, 245419 (2010).

11 W. W. Chow, F. Jahnke, and C. Gies, Light Sci. Appl. 3, e201 (2014).

12 E. Mascarenhas, D. Gerace, M. França Santos, and A. Auffèves, Phys. Rev. A 88, 063825 (2013)

13 S. Strauf, K. Hennessy, M. T. Rakher, Y.-S. Choi, A. Badolato, L. C. Andreani, E. L. Hu, P. M. Petroff, and D. Bouwmeester, Phys. Rev. Lett. 96, 127404 (2006).

14 S. M. Ulrich, C. Gies, S. Ates, J. Wiersig, S. Reitzenstein, C. Hofmann, A. Löffler, A. Forchel, F. Jahnke, and P. Michler, Phys. Rev. Lett. 98, 043906 (2007).

15 C. Ning, IEEE J. Sel. Top. Quantum Electron. 19, 1503604

16 Editorial, Nat. Photonics 11, 139 (2017).

17 P. Gartner, Phys. Rev. A 84, 053804 (2011).

18 R. Loudon, The Quantum Theory of Light, 3rd ed. (Oxford University Press, New York, 2000).
19 M. Orszag, Quantum Optics, 2nd ed. (Springer, Berlin Heidelberg New York, 2008).

20 M. O. Scully and W. E. Lamb, Phys. Rev. 159, 208 (1967)

21 S. Stenholm, Phys. Rep. 6, 1 (1973).

22 J. Ruiz-Rivas, E. del Valle, C. Gies, P. Gartner, and M. J. Hartmann, Phys. Rev. A 90, 033808 (2014)

23 G. S. Agarwal and S. Dutta Gupta, Phys. Rev. A 42, 1737 (1990)

24 Y. Mu and C. M. Savage, Phys. Rev. A 46, 5944 (1992)

25 A. Naesby, T. Suhr, P. T. Kristensen, and J. Mørk, Phys. Rev. A 78, 045802 (2008)

26 E. del Valle, F. P. Laussy, and C. Tejedor, Phys. Rev. B 79, 235326 (2009).

27 M. Richter, A. Carmele, A. Sitek, and A. Knorr, Phys. Rev. Lett. 103, 087407 (2009)

28 E. del Valle and F. P. Laussy, Phys. Rev. Lett. 105, 233601 (2010)

29 H. J. Carmichael, Statistical Methods in Quantum Optics, 2013).ol. 1 (Springer, Berlin Heidelberg New York, 1999).

${ }^{30} \mathrm{G}$. Björk and Y. Yamamoto, IEEE J. Quantum Electron. 27, 2386 (1991).

31 A. Moelbjerg, P. Kaer, M. Lorke, B. Tromborg, and J. Mørk, IEEE J. Quantum Electron. 49, 945 (2013)

32 J. Fricke, Ann. Phys. (NY) 252, 479 (1996).

33 W. W. Chow and F. Jahnke, Prog. Quantum Electron. 37, 109 (2013). 